\title{
Zwischen Fake News und Unterrichtsmaterial - Medien in der geographischen und wirtschaftlichen Bildung
}

\author{
Tagungsbericht Zukunft Fachdidaktik GW, Schlierbach 2018
}

\author{
*herbert.pichler@univie.ac.at, Arbeitsgruppe Fachdidaktik Geographie und wirtschaftliche Bildung, Institut für Geographie und Regio- \\ nalforschung, Universität Wien \\ ** matthias.fasching@univie.ac.at, Studienassistent, Arbeitsgruppe Fachdidaktik Geographie und wirtschaftliche Bildung, Institut für \\ Geographie und Regionalforschung, Universität Wien
}

\begin{abstract}
Dieser Tagungsbericht soll all jenen, die nicht persönlich in Schlierbach anwesend sein konnten, einen Einblick in das Tagungsgeschehen samt vielfältigen Diskussionen, erzielten Erkenntnissen und aufgeworfenen Fragen geben. Zudem dient er auch allen Teilnehmerinnen und Teilnehmern als Anregung zur weiteren Verarbeitung der persönlichen Erlebnisse und Erfahrungen.
\end{abstract}

Bereits zum dritten Mal fand zwischen dem 23. und 25. April 2018 die Tagung ,Zukunft Fachdidaktik GW' im oberösterreichischen Schlierbach statt. Es trafen dort Fachdidaktiker/innen, Lehrer/innen, Studentinnen und Studenten, Interessensvertreter/ innen und Fachwissenschaftler/innen mit Bezug zum Unterrichtsfach GW aus allen Institutionen aller Bildungscluster Österreichs aufeinander. Dies ermöglicht alljährlich einen Austausch und eine gemeinsame Weiterentwicklung. Dazu wurde heuer das Thema ,Medien in der geographischen und wirtschaftlichen Bildung' aufgegriffen und - ganz nach dem Tagungstitel ,Zwischen Fake News und Unterrichtsmaterial - sowohl in einem gesamtgesellschaftlichen, einem wissenschaftlichen als auch in einem unterrichtsspezifischen Kontext beleuchtet.

Veranstaltet wurde die diesjährige Tagung von ,GW-Unterricht - Verein für geographische und wirtschaftliche Bildung' in Zusammenarbeit mit verschiedenen österreichischen Universitäten und Pädagogischen Hochschulen. Das Kernorganisationsteam bestand aus Christian Fridrich, Alfons Koller, Herbert Pichler und Robert Vogler. In der Begrüßung und Tagungseröffnung wurde auch den Sponsoren gedankt, ohne die eine Durchführung bedeutend schwieriger wäre. Besonders hervorzuheben ist wieder die $\mathrm{AK}$ Wien als Hauptsponsor, die es uns ermöglicht hat,

Wir bedanken uns bei Christiane Hintermann und Robert Vogler für ihre Unterstützung sowie bei Christian Fridrich, Lars Keller und Johannes Wimmeder, die uns Fotos zur Verfügung gestellt haben. hochkarätige Keynote Speaker und Referentinnen und Referenten - beispielsweise die Expertin für digitale Medien, Ingrid Brodnig, oder den Anchorman der ,Zeit im Bild 2‘ Armin Wolf - einzuladen. Weitere Förderer/innen und Unterstützer/innen waren die AK Salzburg, die Industriellenvereinigung, sowie das Institut für Fort- und Weiterbildung der PH Linz.

Der zugespitzte Titel der Tagung mag bei manchen Fragen aufgeworfen haben: Was haben Fake News mit Unterrichtsmaterialien zu tun? Der erste Gedanke: Hoffentlich herzlich wenig - ein Schulbuch, ein Arbeitsblatt und selbst eine Online-Lernplattform sollten keine Fake News verbreiten und keine falschen Tatsachen, sondern möglichst aktuelle Zugänge und Perspektiven sowie gesichertes Wissen bereitstellen. Freilich eröffnen sich zahlreiche Verbindungen zwischen Fake News, Schulunterricht und Unterrichtsmaterialien. Wir sind dazu verpflichtet, GW-Unterricht so zu gestalten, dass die Schüler/innen für ihr privates, berufliches und öffentliches Leben entscheidungsund handlungsfähig werden (vgl. BMBWF 2017 und BMBWK 2000). Dazu gehört eben auch die Auseinandersetzung mit dem aktuellen und gesellschaftlich wichtigen Diskurs zur Entwicklung verschiedener Medien und dem kompetenten und kritischen Umgang mit ihnen. Dieser hat zentrale Bedeutung für die Entscheidungs- und Handlungsfähigkeit unserer Schüler/ innen, Medienkompetenz bestimmt in hohem Maß die Möglichkeiten ihrer aktuellen und zukünftigen Beteiligung an gesellschaftlichen und wirtschaftlichen Prozessen. Die GW-Fachdidaktik und der in der 
Schule realisierte GW-Unterricht haben idealerweise die Bedeutung der Medien für die Konstruktion von Weltbildern und Wertvorstellungen längst erkannt, der Erwerb von Urteils- und Kritikfähigkeit, das Prinzip der Mehrperspektivität, die Kritische Medienkompetenz, das Basiskonzept Kontingenz und die Politische Bildung sind fixer Bestandteil in den Lehrplänen des Faches in den verschiedenen Schultypen und in der gemeinsamen (Aus-)Bildung von GWLehrerinnen und -Lehrern an Universitäten und Pädagogischen Hochschulen. Auch konstruktivistische Lerntheorien stellen eine Verbindung zwischen dem darauf aufbauenden GW-Unterricht und einer Reflexion der Mediennutzung her. Nicht zuletzt verlangen die beiden fächerübergreifenden Unterrichtsprinzipien der Politischen Bildung und der Medienbildung eine Aufarbeitung des Tagungsthemas in allen Unterrichtsfächern: Wie wirken Medien? Welche Wirkungen sind abschätzbar, welche sind nicht (mehr) steuerbar? Wer beeinflusst die Mediengestaltung? Welche Rollen kommen klassischen Massenmedien, welche dem Social Web zu? Wie reagieren die Konsumentinnen und Konsumenten und die Prosumer/innen auf ihre Medien? Welche Fähigkeiten sind für „medienkompetente Personen " bedeutend?

Neben dem kritischen Hinterfragen der Konstruktion und Nutzung von Medien im Alltag wurde ein genauerer Blick auf jene Medien geworfen, die traditionell im GW-Unterricht verstärkt eingesetzt werden: Schulbuch, Arbeitsblatt, Atlas, Karte, Onlinequellen etc. Dadurch entwickelt sich also eine zweite Diskussionsebene, die sich mit ersten Leitgedanken für ein ,Schulbuch(?) 2030' auseinander setzte: Wie muss oder soll ein zeitgemäßes und zukunftsorientiertes Unterrichtsmedium gestaltet sein, was soll ein aktuelles Schulbuch beinhalten? Wo liegen die Stärken und Schwächen des Mediums Schulbuch? Ist das Format des Schulbuches überhaupt noch zeitgemäß oder prinzipiell zeitlos? Welche anderen, alternativen Unterrichtsmedien und -materialien können oder sollen Einzug in den GW-Unterricht halten? Wie wird auch die Anfertigung von Unterrichtsmaterialien durch den persönlichen Medienkonsum beeinflusst? Auch zu diesen Fragen kam es auf der dritten Tagung, $\mathrm{Zu}$ kunft Fachdidaktik GW' zu intensiven und spannenden Diskussionen und Gesprächsrunden.

Zusammenfassend wurden in diesem politischen und wirtschaftlichen Spannungsfeld aktuelle fachdidaktische Perspektiven, aber auch die aktuelle Situation der Schulbücher und Unterrichtsmedien analysiert. Der gesellschaftliche Wandel durch die digitalen Medien, seine Rezeption in Unterricht und Schule wurden diskutiert und daraus ableitbare Handlungsmöglichkeiten für Lernende und Lehrende entwickelt.
Dazu wurde ein abwechslungsreiches Programm, gespickt mit Expertinnen- und Expertengesprächen, Exkursionen und Inputs der Tagungsteilnehmer/innen, angeboten. Daneben blieb jedoch genügend Zeit und Raum für den Austausch abseits des Tagungsthemas, die Vernetzung zwischen einzelnen Akteurinnen und Akteuren, für Projektvorstellungen von (Jung-)Forscher/innen anhand einer Poster-Session, sowie eine künstlerische Aufarbeitung des Tagungsthemas durch das Kabarettduo ,Gerafi' als Abendprogramm. Die folgenden Ausführungen sollen zentrale Aspekte der Keynotes, Vorträge und der daran anknüpfenden Gespräche widerspiegeln.

\section{Keynotes und Expertinnen- und Exper- tengespräche}

Wieder konnten im Zuge der Tagung, Zukunft Fachdidaktik GW' hochkarätige Expertinnen und Experten für Vorträge und Gespräche gewonnen werden. Die folgenden Ausführungen sollen Aspekte der Vorträge und der daran anknüpfenden Gespräche wiederspiegeln.

\subsection{Digitale Medien im schulischen Kontext}

Nach der Tagungseröffnung durch das Organisationsteam und den einführenden Worten des Vertreters der Arbeiterkammer Wien Richard Meisel sowie von Tina Dworschak als Vertreterin der Industriellenvereinigung Österreich, führte Andreas Riepl, Leiter des Bundes- und Koordinationszentrums eEducation Austria des Bundesministeriums für Bildung, Wissenschaft und Forschung, mit der Keynote „Digitale Medien im schulischen Kontext " in das Tagungsthema ein.

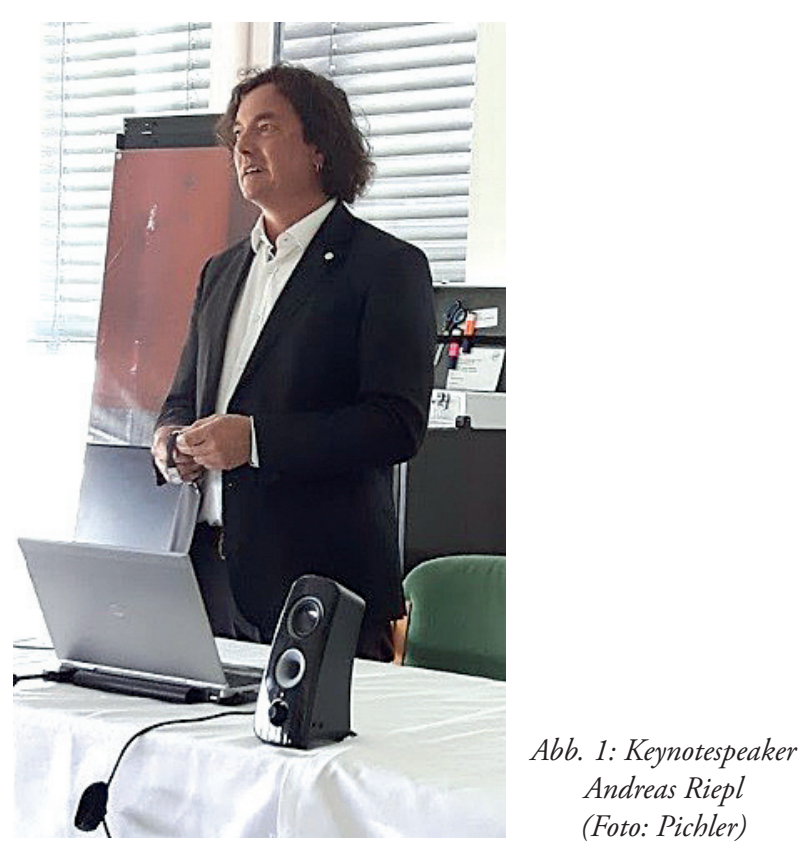


Basierend auf der ,JIM'-Studie (Jugend, Information, (Multi-)Media) von 2017 zeichnete Riepl ein Bild von der Bedeutung digitaler Medien für Jugendliche. So besitzen $97 \%$ der Jugendlichen in Deutschland ein Smartphone, am häufigsten nutzen sie die Internetdienste YouTube und WhatsApp, vergleichbar ist die Durchdringung der Lebenswelt der Jugendlichen in Österreich. Internet und Smartphone werden von rund $90 \%$ der Jugendlichen täglich genutzt. Neben den als positiv einzustufenden Möglichkeiten des $\mathrm{Zu}$ griffs auf Information und der unbeschränkten Kommunikation birgt die Nutzung aber auch zahlreiche bekannte Gefahren. So geben rund 4 von 10 Jugendlichen an, dass in ihrem Bekanntenkreis schon einmal jemand Opfer von Mobbing oder Bullying via Handy oder Internet wurde. Auch die Speicherung von $\mathrm{Da}-$ ten im Zuge der Nutzung muss kritisch reflektiert und transparent gemacht werden. Jugendliche hinterlassen wie alle Nutzer/innen im Internet bleibende Spuren in Form von Kommentaren, Beiträgen, Bildern, Likes etc. Die daraus oft unreflektiert konstruierbare oder konstruierte, Online-Identität' kann jederzeit von anderen Personen - zum Beispiel zukünftigen Arbeitgeberinnen und Arbeitgebern oder zum Zweck personalisierter Werbung - ausgeforscht und überprüft werden. Dieses Spannungsfeld der Chancen, Möglichkeiten und Gefahren begründet die Notwendigkeit einer umfassenden Auseinandersetzung mit eEducation und der digitalen Bildung in der Schule.

Die Dringlichkeit der Auseinandersetzung wird laut Riepl durch Analysen des IKT-Einsatzes im Unterricht unterstrichen. Während Lehrer/innen den IKT-Einsatz im Unterricht überwiegend positiv bewerten, vergeben die Schüler/innen dafür die schlechtesten Noten. Gleichzeitig glauben Lehrer/innen auf Grund mangelnder Infrastruktur oder Qualifikation die hohen Erwartungen der Schulleitungen und Schulbetreiber/innen nicht erfüllen zu können. Nicht überraschend kann festgestellt werden, dass Lehrer/ innen mit fortgeschrittenen fachdidaktischen und methodischen Kompetenzen auch die größte Vielfalt im angewandten IKT-Einsatz zeigen. Als Veranschaulichung der unterschiedlichen Reflektiertheit und

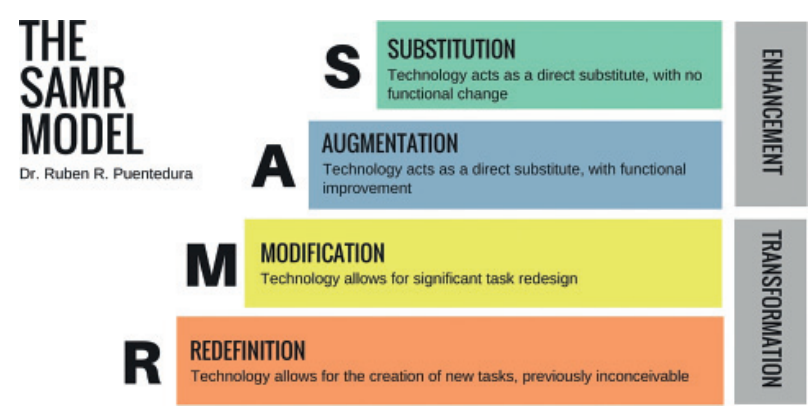

Abb. 2: Das SAMR-Modell (Quelle: https://upload.wikimedia.org/ wikipedialcommons/5/5e/The_SAMR_Model.jpg)
Komplexität und damit zur Differenzierung des IKTEinsatzes kann das, SAMR'-Stufenmodell dienen. Dabei wird unterschieden, ob Medien und ihre Einsatzmöglichkeiten im Zuge der Digitalisierung schlicht ausgetauscht (substituiert), erweitert (augmentiert), modifiziert oder neu definiert (Redefinition) werden (siehe Abb. 2).

Die eEducation-Initiative des Bundesministeriums für Bildung, Wissenschaft und Forschung versucht, bestehende schulische Initiativen zu vernetzen und weitere Schulen mittels Anreizsystemen und positiver Verstärkung dazu zu bewegen, digitale Medien auf verschiedenen Ebenen im schulischen Kontext zu testen und Erfahrungen zu teilen. Dies inkludiert nicht nur digitale Medien im Unterricht und innovative Lerntechnologien einzusetzen, sondern auch die Unterstützung von Schulentwicklungsprozessen und schulübergreifenden Kooperationen. Digitale Kompetenzen sollen hierbei nicht nur bei Schülerinnen und Schülern, sondern auch bei Lehrerinnen und Lehrern gefördert werden. Die eEducation-Initiative versucht dabei, Schulen und Lehrer/innen möglichst laufend am Ball zu halten, indem für den Erhalt des Status im eEducation-Netzwerk von einer Schule jedes Jahr Projekte angegeben werden müssen.

Digitale Bildung soll nach den Plänen der eEducation-Initiative möglichst flächendeckend und möglichst umfangreich nicht nur bezüglich Zielpersonen (Lehrer/innen, Schüler/innen, Schulleiter/innen, etc.), Methodiken (Einsatz von Whiteboards, digitalen Lernmodulen, etc.) sondern auch im Bereich der Inhalte (Umgang mit Mobbying im Internet, Creative Commons, etc.) umgesetzt werden. Andreas Riepl vertrat glaubwürdig die Position, dass es nicht um eine blinde Etablierung von Technologien gehe, neben den digitalen gelte es bei Lehrenden und Lernenden im thematischen Feld auch soziale und personale Kompetenzen zu entwickeln.

\subsection{Klassische Medien, Social Media und kritische Mediennutzung}

Robert Vogler von der Universität Salzburg eröffnete diese Session mit einem kurzen pointierten Input. Ausgehend von der Prämisse, dass einerseits „nur Weniges eine so große Bedeutung für die Strukturen der Gesellschaft hat wie das jeweils dominierende Verbreitungsmedium [von Informationen]" (Baecker 2007: 7) und wir andererseits mit der Digitalisierung gerade am Beginn des nächsten Leitmedienwechsels stehen, der in seiner Durchschlagskraft nur mit dem Aufkommen des Buchdrucks und all seinen Folgen (z.B. der Aufklärung) zu vergleichen ist, ließ er die Zuhörer/innen zunächst im Dunkeln. Denn bereits auf seiner zweiten Folie gestand er: „Wir wissen nicht 


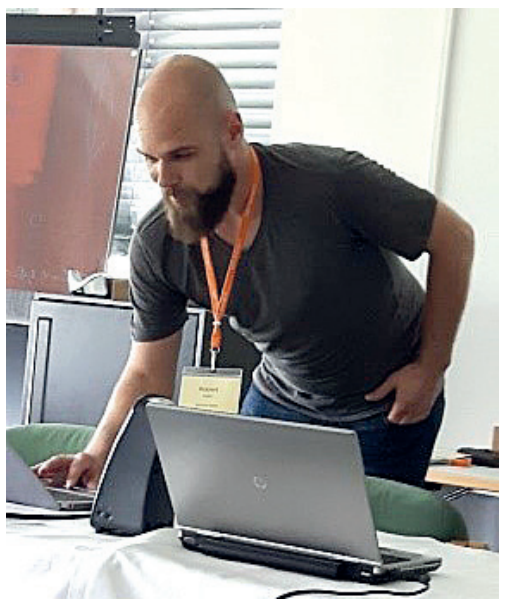

Abb. 3: Robert Vogler, Universität Salzburg (Foto: Pichler)

im Geringsten, welche weitreichenden Folgen die Etablierung einer neuen Mediosphäre haben wird!“.

Anschließend skizzierte er einige, bereits jetzt beobachtbare Tendenzen, wie sich die neuen medialen $\mathrm{Zu}-$ gänge im Alltag bemerkbar machen und diesen zum Teil massiv transformieren. Dabei ist die inzwischen ubiquitäre Verfügbarkeit nahezu sämtlicher Informationen noch die trivialste, da sichtbarste Neuerung: Wissen wird somit zu einem fluiden Konzept. Doch die aktuellen Entwicklungen gehen weit über das simple, just get it online ' hinaus, da es nicht nur um Zugang, sondern vor allem um Vernetzung geht. Das zentrale Schlagwort heißt hier ,Plattform'. Ob Amazon, Facebook, Google oder unsere (mobilen) Betriebssysteme: Gemeinsamkeit all dieser, Big Player' ist, dass sie nicht Inhalte zur Verfügung stellen, sondern als Schnittstellen zwischen uns und dem Rest der Welt funktionieren und damit mächtig sind. Dies sind sie vor allem dann, wenn sie basierend auf unserem bisherigen Online-Verhalten - und in jüngster Vergangenheit eben zunehmend über intelligente Algorithmen - intransparent beeinflussen, welche Inhalte wir sehen und welche nicht. Konsequenterweise bilden sich somit (teilweise ohne dass wir es merken) recht schnell Sub-Öffentlichkeiten und Filterblasen: Wir sehen vermehrt Inhalte und Meldungen, die in unser Weltbild passen. Damit ist dann das Tor für Fake News und ,alternative Fakten' geöffnet. All diese Phänomene werden insofern noch brisanter, da die Informationsflüsse sich auch strukturell geändert haben: Wir bekommen zunehmend auf uns zugeschnittene Informationen, ohne aktiv nach diesen gesucht zu haben!

Darüber hinaus lassen sich noch andere Phänomene entdecken, die weniger in der Granularisierung von Informationen fußen, sondern eine direkte Konsequenz der schieren Informationsflut sind, die täglich auf uns einstürmt. Einerseits geht die wachsende Ubiquität von Informationen mit einer sinkenden Historizität derselben einher: Die Story von heute haben wir morgen bereits vergessen, weil es neue Stories gibt. Andererseits - und das bereitet Grund zur Sorge - sind besonders jene Stories, die uns emotional berühren, besonders erfolgreich. Konsequenz ist, dass die Stories schnell vergessen werden, die dahinterstehenden Narrative aber bleiben (z. B. das hartnäckige „Die Flüchtlinge sind an allem Schuld“-Motiv). Kombiniert man dies mit den oben angesprochenen Filterblasen und Sub-Öffentlichkeiten, ist Vorsicht geboten; vor allem deswegen, weil Plausibilität immer weniger als Gradmesser einer Information taugt. Mit diesen Effekten geht direkt die Genese von Weltbildern einher und spätestens dann sind all diese Phänomene auch in hohem Maße für (geographische) Bildung relevant.

All dies sind nur Nuancen, die andeuten, wie sich unsere Medienlandschaft und damit unser Informationskonsum bereits verändert hat und noch verändern wird, resümierte Vogler abschließend. Die (nahe) Zukunft bleibt spannend! Damit bot er schließlich einen fließenden Übergang zur Diskussion mit Armin Wolf, der zweifelsohne eine breite Expertise für beide Welten, die alte und die neue Medienlandschaft, aufweisen kann. Armin Wolf, österreichischer Journalist und Anchorman der ,Zeit im Bild 2', wurde live via Videotelefonie in die große Diskussionsrunde zugeschalten. Das Gespräch mit dem frischgebackenen GrimmePreisträger unter dem Fokus „Zwischen Facts und Fake? News in klassischen Medien und Social Media " und wurde durch die Fragen der Tagungsteilnehmer/ innen strukturiert.

Auch Wolf betonte dabei die Veränderung der Mediennutzung bei jungen Menschen und führte in Hinblick auf die bekannte Altersstruktur des Nachrichtenpublikums aus, ob es Nachrichtensendungsformate wie jenes der ZiB 2 in Zukunft überhaupt noch brauchen oder geben würde. Ausschlaggebend dafür,
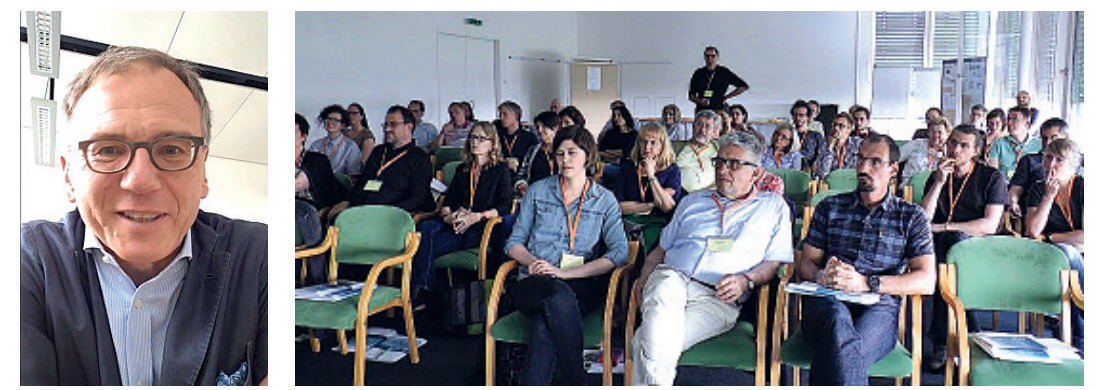

Abb. 4: Screenshot der Videoschaltung mit Armin Wolf(ORF) und Diskutantinnen und Diskutanten im Plenum (Foto: Wimmeder) 
ob Jugendliche in Gegenwart und Zukunft auch klassische Medien nutzen, sei seiner Meinung nach das Vorbild der Eltern. Ein junger Mensch würde hier eher zu einer Zeitung greifen, wenn dieses Medium aus dem eigenen Elternhaus bekannt ist.

Gleichzeitig entstünden laufend neue Medienformate und digitale Medien, welche aufgrund der schnellen Erzielung einer hohen Reichweite auch sofort in den Konkurrenzkampf mit etablierten Medien treten. In dieser Entwicklung sieht Wolf auch die Problematik der Emotionalisierung und der Fake News verankert: Während früher die eigenen Ansichten nur im persönlichen Umfeld - am Stammtisch - geteilt werden konnten, ist die Erstellung eines privaten Blogs für viele Menschen keine große Hürde mehr. Jedes Gedankengut, von aufklärerisch bis extremistisch, von gut belegbar bis wissentlich falsch, kann dadurch deutlich leichter einem breiteren Publikum mit vergleichsweise geringem Aufwand zugänglich gemacht werden.

Auch der politische Einfluss auf und von Medien habe dadurch zugenommen. Einzelne politische Parteien können nun ebenso mit klassischen Medien in der digitalen Welt konkurrieren und über eigene Kanäle ihre Klientel bedienen und somit besser kontrollieren, welche Informationen in welcher Weise nach außen getragen werden. Dadurch, dass das Erscheinen in Zeitungen oder Nachrichtensendungen zur Positionierung von Statements und politischen Vertreterinnen und Vertretern nicht mehr zwingend notwendig sind, um Menschen zu erreichen, wird die Rolle der klassischen Medien zusätzlich geschwächt. Trotzdem, mutmaßt Wolf, würde eine potentielle Volksabstimmung über die Abschaffung der österreichweiten Rundfunkgebühr wie in der Schweiz vermutlich zugunsten der Gebühr ausfallen. Problematischer sieht er eine mögliche Eingliederung des ORF-Budgets in das Staatsbudget, wodurch die Bundesregierung direkten Zugriff auf die Gelder für den öffentlich-rechtlichen Rundfunk und damit verbunden noch mehr Einflussmöglichkeiten auf diesen gewinnen würde.

Allgemein werde es laut Wolf erforderlich, die Entwicklungen rund um die Medienlandschaft kritisch zu hinterfragen und Medien auch reflektiert zu kon- sumieren. Generell sollten digitale Medienplattformen ebenso reguliert sein und einer Form von Pressekodex unterliegen. Junge Menschen sollten in der Schule zudem vermehrt auf die Vor- und Nachteile von klassischen und neuen Medien aufmerksam gemacht und diesbezüglich sensibilisiert werden.

\subsection{Fake News und Förderung von Medien- kompetenz}

Mit dem Vortrag „Fake News in Politik und Wirtschaft - Wie Lehrer/innen die Medienkompetenz fördern können" gab Ingrid Brodnig, Journalistin und Expertin für digitale Themen, weitere Einblicke in problematische Entwicklungen der digitalen Welt und konkrete Handlungsvorschläge für Lehrpersonen. Brodnig erörterte an konkreten, gut dokumentierten Beispielen, wie und wo Fake News als Mittel politischer Stimmungsmache eingesetzt werden. Grundsätzliche seien falsche Nachrichten nach der Intention der Verfasser/innen zu differenzieren. Hoaxes sind etwa Falschmeldungen, die als Scherz gedacht sein können und über soziale Medien geteilt werden und virale Verbreitung finden können. Andere entstehen durch Gerüchte und schlechte Recherche. Unabhängig von der Absicht können sie eine vergleichbare Wirkung unter den Mediennutzer/innen entfalten. Besonders dann, wenn Fake News auch bewusst von seriös anmutenden Internetseiten in Umlauf gebracht werden, die nicht auf den ersten Blick als Propagandainstrumente erkannt werden können. Verstärkt werden die Effekte der Verbreitung von Fake News dadurch, dass vor allem provokative, emotionalisierende, oder ,faktastische ${ }^{6}$ Nachrichten von den Internetznutzerinnen und -nutzern angeklickt und gelesen werden und auf der anderen Seite eine breitenwirksame Widerlegung von falschen Tatsachen schwierig zu realisieren ist.

Für Lehrpersonen bedeutet dies, dass Schulunterricht, der die Medienkompetenz von jungen Menschen fördern will, deshalb berücksichtigen muss, wie Teenager prinzipiell das Netz und seine Angebote nutzen, warum falsche Nachrichten so erfolgreich sind und wie Aufklärung geschafft werden kann. Eine

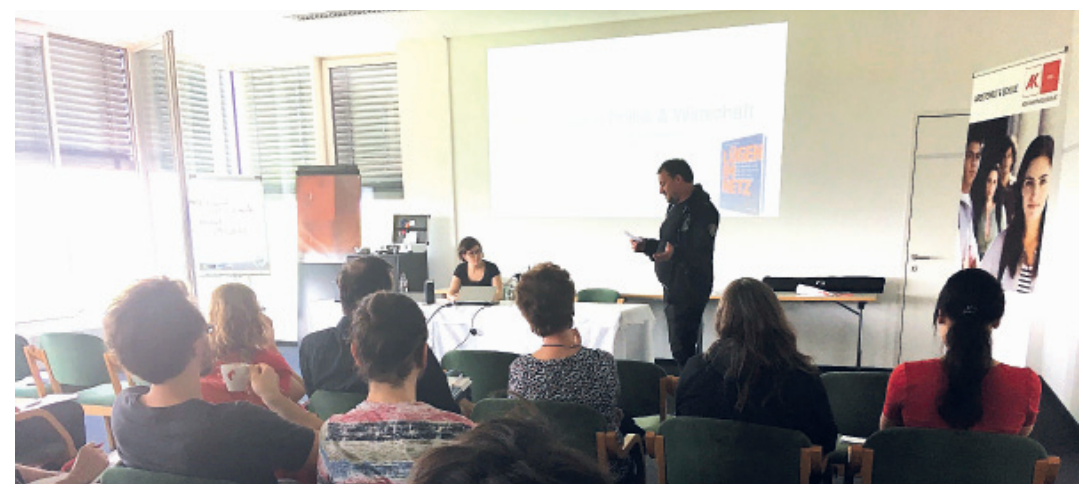

Abb. 5: Begrüßßung von Ingrid Brodnig durch Christian Fridrich (Foto: Keller) 
wichtige Voraussetzung für die Medienkompetenz von Jugendlichen in Bezug auf das Erkennen und die routinierte Einordnung zuverlässiger Nachrichten oder Fake News ist, dass man selbst Fakten recherchieren und überprüfen kann. Dabei kann zum Beispiel auf die Seite des Vereins ,Mimikama' verwiesen werden, welcher sich der Aufklärung des Missbrauchs des Internets zur Verbreitung falscher Nachrichten verschrieben hat. Allgemein gibt Brodnig drei Tipps, um Falschmeldungen (im Unterricht) zu erkennen: Es sollte auf ausgelöste Gefühle geachtet, veröffentlichten Bildern grundsätzlich misstraut und konsequent Quellenkritik geübt werden. So können mittels Google-Bildersuche beispielsweise auch Bilder hochgeladen oder Links eingefügt werden, um herauszufinden, woher Bildquellen ursprünglich stammen beziehungsweise in welchen Zusammenhängen das Bild noch (fälschlich) verwendet wird. Weiters kann in Suchmaschinen die Richtigkeit einer Nachricht leichter recherchiert werden, indem an die Sachverhaltsbeschreibung in der Suchleiste das Wort ,Faktencheck' angefügt wird. Auch Quellen lassen sich relativ schnell und effizient recherchieren. Schlussendlich sollten natürlich auch alle Formen von Statistiken bezüglich ihrer grafischen Aufbereitung kritisch beleuchtet werden.

\section{Exkursionen}

Als ein produktiver und wesentlicher Bestandteil der Arbeitstagung, Zukunft Fachdidaktik GW' haben sich Exkursionen zum jeweiligen Tagungsthema erwiesen. So teilten sich am zweiten Tag die rund 40 Teilnehmer/innen in drei Kleingruppen auf und besuchten die ,Oberösterreichischen Nachrichten', die ,EduGroup' oder das ,Ars Electronica Center' in Linz, um herauszufinden, welche konkreten Spuren der digitale mediale Wandel in verschiedenen Bereichen der Medien- und Bildungsmedienwelt hinterlässt.

\subsection{OÖN}

Nicht selten werden Artikel und Beiträge aus Zeitungen und Fernsehen im Unterricht eingesetzt. Aktualitäts- und Lebensweltorientierung verlangen, realweltliche Themen miteinzubeziehen. Dabei stellt sich in der Debatte um Fake News und neue Medien die Frage, inwieweit den klassischen Medien noch vertraut werden darf, worauf beim Konsum solcher geachtet werden sollte und wie sie mit einem möglichen radikalen Umbruch in der Medienlandschaft umgehen.

Chefredakteur Gerald Mandlbauer empfing eine Gruppe im neuen Verlagsgebäude der Oberösterreichischen Nachrichten und nahm sich dankenswerterweise Zeit für eine offene Diskussion. Für das
Tagungsthema war der Besuchspunkt deswegen ausgewählt worden, da erst vor kurzem die Redaktionen der Printausgabe der OÖN sowie die Online-Redaktion in einem modernen Newsroom zu einer gemeinsamen Redaktion zusammengelegt worden waren. So konnte live beobachtet werden, wie in einer hierarchischen Redaktionsstruktur das klassische Medium Tageszeitung und gleichzeitig das hochfrequente OnlineProdukt gestaltet wird. Eindrucksvoll erwiesen sich etwa die auf der großformatigen digitalen Newswall in Echtzeit angezeigten Nutzerzahlen und Verweildauern der erfolgreichsten Beiträge der Online-Ausgabe. Gossip, Lokalmeldungen und Verbraucher/innentipps werden deutlich häufiger angeklickt und gelesen als überregionale oder globale Nachrichten über Gesellschaft, Wirtschaft oder Politik. Mandelbauer erörterte an diesem Beispiel die Herausforderung des klassischen Journalismus mit Qualitätsanspruch in direkter Konkurrenz zu Gratisblättern und digitalem Boulevard. Die Online-Redaktion platziert trotzdem als relevant eingestufte Nachrichten prominent auf der Online-Seite, vergleichsweise unbedeutend angesehene Meldungen werden eher versteckt platziert. Freilich hat die Redaktion die Nutzerzahlen ständig vor Augen und den Auftrag auf abfallendes Interesse sofort durch eine Umgestaltung zu reagieren.

Als besonders spannend erwies sich die Ergänzung um die Perspektive des Leiters der Marketingabteilung der OÖN. Dies machte deutlich, dass etliche journalistische Entscheidungen (Blattlinie und journalistischer Qualitätsanspruch, die Reihenfolge der Themen, der ,Bücher' in der Zeitung, die Begrenzung von Werbemöglichkeiten etc.) auch negative Konsequenzen für den Absatz und den Werbeerlös der Zeitung haben können. Als besonders prekär erscheint der vonseiten der Chefredaktion erhobene Anspruch auf völlige Unabhängigkeit einer Zeitung mit regionalem Schwerpunkt von der Landespolitik bei genauerer Betrachtung der zahlreichen Regional- und Lokalausgaben der Zeitung (z.B. „Braunauer Warte"). Wie kann hier von einem unabhängigen Verhältnis zu lokaler oder regionaler Politik und Wirtschaft ausgegangen werden, wenn die Finanzierung von Lokal- und Regionalausgaben unter anderem von Einnahmen durch Werbung oder Spezialbeilagen von Gemeinden, Verbänden oder Betrieben abhängig ist? Das Lokale und Regionale ist der Wettbewerbsvorteil von Regionalzeitungen wie den Oberösterreichischen Nachrichten, der offenbar auch im Zeitalter der digitalen Medien Bestand hat. Doch ähnlich wie in vielen digitalen Medien sind dabei die Grenzen zwischen Information und Werbung, zwischen Journalismus und Public Relations manchmal schwer erkennbar.

Die zentralen Erkenntnisse des Besuchs in der Redaktion der OÖN wurden in der Präsentation im 
Rahmen der Tagung am abschließenden Tag von den Teilnehmerinnen und Teilnehmern in eine theatrale Vermittlungsform verpackt: Chefredakteur und Marketingverantwortlicher beraten live auf offener Bühne über die Verwertbarkeit von laufend eintreffenden Nachrichten - in Form von Kolleginnen und Kollegen, die sich als jeweilige Nachrichten über konkrete Ereignisse von royalem Gossip über nur lokal bedeutenden Kleinstereignissen bis hin zu weltpolitischen Nachrichten vorstellen - und geben damit einen Einblick in das Spannungsfeld zwischen journalistischem Anspruch und ökonomischen Verwertungslogiken in klassischen Printmedien im Vergleich zu OnlineMedien.

\subsection{EduGroup}

Bei einer Erweiterung des ,klassischen ' Medien-Begriffes kann festgestellt werden, dass im GW-Unterricht zahlreiche Formen von Medien eingesetzt werden. Auch diese Medien erfahren eine Weiterentwicklung, so entstehen immer mehr Lernplattformen, Lernmodule und ähnliches. Inwieweit sind solche Angebote schon ausgereift, wie können sie im Unterricht eingesetzt werden und (warum) sind sie vielversprechender als bisherige Medien?

Um dies herauszufinden verbrachte eine Exkursionsgruppe einen Vormittag in der Redaktion der EduGroup, einem Anbieter einer bislang in Österreich konkurrenzlosen Plattform für Lehrund Lernmaterialien. Das Angebot der digital zur Verfügung gestellten Materialien ist beeindruckend: ÜberdieSammlungklassischer Lehrfilmehinausstehen digitale Archive wie die umfangreiche Audiothek oder die Cinemathek zur Nutzung bereit. Die EduGroup sammelt und erstellt Medienpakte und Lernpakte, von klassischen Unterrichtsmaterialien und Arbeitsblättern bis hin $\mathrm{zu}$ interaktiven Lernumgebungen für alle Altersstufen des Schulsystems. Darüber hinaus werden Lehrpersonen über Veranstaltungen, Fortbildungen und schulrelevante Wettbewerbe informiert sowie beim Ausprobieren von technischen Innovationen im Unterricht unterstützt. Die Mitarbeiter/innen betonten das angenehme Arbeitsumfeld und die Vorzüge des Arbeitsfeldes im Zusammenspiel zwischen Kreativen und Programmierer/innen. Kritisch kann angemerkt werden, dass das Erstellen und Bereitstellen von Unterrichtsmaterialien und Medien nicht nur eine Frage der Kreativität sowie der technischen Umsetzbarkeit (etwa Ausschöpfen der Möglichkeiten digitaler Medien) bedeutet. Die didaktische Perspektive und kritische Betrachtung der gewählten Methodiken erscheint ausbaufähig, hier eröffnet sich ein mögliches Feld verstärkter Kooperation mit der GW-Fachdidaktik sowie mit qualifizierten
Schulpraktikerinnen und -praktikern. Ein kleiner Wehrmutstropfen muss abschließend angemerkt werden, da die EduGroup maßgeblich von der oberösterreichischen Landesregierung finanziert wird, steht der volle Umfang der Nutzungsmöglichkeiten der Plattform nur für Lehrpersonen aus Oberösterreich zur Verfügung.

\subsection{Ars Electronica Center}

Schlussendlich beeinflussen sich Gesellschaft, Medien und Technologie wechselseitig. Der GW-Unterricht soll einen Beitrag dazu leisten, junge Menschen für die Anforderungen der Zukunft vorzubereiten und ihnen ein Rüstzeug mitzugeben, in einer zukünftigen Welt verantwortungsbewusst entscheiden und handeln zu können. Dazu sollten jedoch die Fragen gestellt werden, in welche Richtung sich Gesellschaft, Medien und Technologien entwickeln, wie ein Leben in der Zukunft aussehen könnte und welche Möglichkeiten und Gefahren damit verbunden sein werden.

Das Ars Electronica Center, das sich selbst als Museum der Zukunft versteht, bietet seinen Besucherinnen und Besuchern interaktive Ausstellungsformate am Schnittpunkt von Kunst, Technologie und Wissenschaft. Wie Menschen durch technologische Entwicklungen ihre Lebensumwelt wahrnehmen, verändern oder darstellen, ist ein zentrales Thema der Ausstellungen. Die Teilnehmer/innen der Exkursionsgruppe wurden durch zwei aktuelle ,Ausstellungen' geführt. Im Deep Space können Bildwelten in 8K Auflösung projiziert werden, Gigapixelfotografien, Zeitraffervideos oder 3D-Visualisierungen von nationalen und internationalen Kulturstätten und Kulturschätzen zählen zum Repertoire. Die Ausstellung, Neue Bilder vom Menschen' lädt zum einen zu einer Auseinandersetzung mit Entwicklungen in den Bereichen von Virtual, Mixed und Augmented Reality ein und hinterfragt zum anderen, (z. B. im so genannten BrainLab)

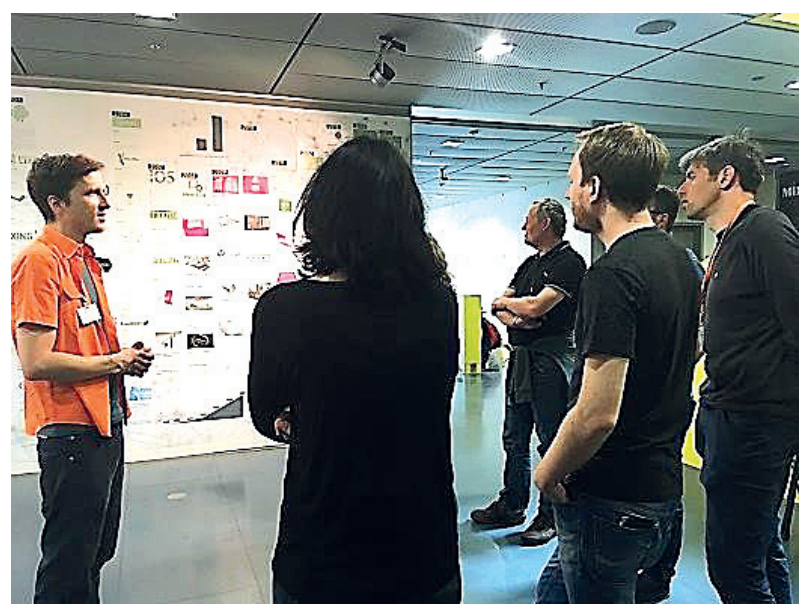

Abb. 6: Impressionen der Exkursionen: Ars Electronica Center (Foto: Keller) 
wie wir unsere Umwelt wahrnehmen. Was ist wirklich, was ist Täuschung, was ist fake? Diese zentralen Fragen wurden auch im letzten Programmpunkt im Ars Electronic Center angesprochen, einem Vortrag zum Thema Fake News und soziale Medien, der inhaltlich viele Anknüpfungspunkte an die Keynotes und Expertinnen- und Expertengespräche der Tagung ermöglichte.

\section{Diskussionen - Austausch - Unterhaltung}

Die Schlierbach-Tagung 2018 ermöglichte auch umfangreiche Diskussionen und einen Austausch unter den Teilnehmerinnen und Teilnehmern. Möglichkeiten boten hierfür insbesondere eine Podiumsdiskussion zur aktuellen Rolle und Zukunft von Unterrichtsmaterialien und eine Vorstellung verschiedener Forschungsarbeiten und Projekte abseits des Tagungsthemas. Zusätzlich sorgten zwei Kabarettisten für die humorvolle Betrachtung der Thematik und einen Blick über den Tellerrand hinaus.

\subsection{Podiumsdiskussion „Medien als GW-Buch - GW-Buch als Medium“}

Einen weiteren Höhepunkt der Tagung versprach die Thesendiskussion mit Vertreterinnen und Vertretern von Schulbuchverlagen, Stakeholdern im Bereich der wirtschaftlichen Bildung und Anbietern von Unterrichtsmaterialien sowie der Fachdidaktik GW zur Gegenwart und Zukunft des Mediums Schulbuch. Unter der Moderation von Sandra Stieger (Universität Salzburg) und Christiane Hintermann (Universität Wien) diskutierten die Teilnehmer/innen Lukas Birsak (Hölzel Verlag), Iris Blatterer (Westermanngruppe), Tina Dworschak (Industriellenvereinigung), Richard Meisel (AK Wien), Herbert Pichler (Universität Wien), Robert Vogler (Universität Salzburg), Veronika Weidenholzer (Veritas). Im Publikum befanden sich weiters eine zweistellige Zahl von aktiven Schulbuchautorinnen und -autoren. Aus gesundheitlichen Gründen abgesagt hat leider die für Schulbuchapprobationen zuständige Ministerialrätin Sonja Hinteregger-Euller (BMBWF).
In der Diskussion wurden u. a. die ökonomischen und legistischen Sachzwänge dargelegt, unter denen Schulbuchverlage und Schulbuchautorinnen und -autoren arbeiten, dies verhindere allzu innovative Schulbuchprojekte. Ein weiterer innovationshemmender Faktor liegt wohl auch in der beobachtbaren konservativen Bestellpraxis der Lehrpersonen, die ,Altbewährtes' den neuen Schulbuchkonzepten vorziehen, da diese adaptierte Unterrichtskonzepte nach sich ziehen würden. Von Seiten der Fachdidaktiker/innen wurde darüber hinaus die nach wie vor dominierende Rolle des Schulbuchs bei der Unterrichtsplanung und -realisierung von Lehrpersonen kritisiert - Stichwort: geheimer Lehrplan - eine Kritik, die Forderungen an die Lehramtsausbildung sowie die Fortbildung nach sich zieht. Vertreter/innen der Schulbuchverlage stellten das Schulbuchangebot als modern, vielfältig und multimedial dar und sehen das Schulbuch auch im 21. Jahrhundert gut aufgestellt. Die Analyse der Gegenwart der GW-Schulbücher und notwendiger Relaunches oder Neuerfindungen von zukunftsfähigen Begleitmedien für Unterricht fiel durchwegs kontrovers aus. Uneinigkeit zwischen Verlagen und Teilen der Schulbuchautorinnen und -autoren auf der einen Seite, Fachdidaktiker/innen und der Vertreterin der Industriellenvereinigung auf der anderen Seite herrschte etwa bezüglich der Vision, welche Aufgaben unterrichtsunterstützende Medien in Zukunft übernehmen sollen und welche Anforderungen sich daraus ergeben. Von Verlagsseite wird das Schulbuch als Konkretisierung des Lehrplans, nötiger Leitfaden für zeitlich und organisatorisch belastete Lehrpersonen, als Kanon des wesentlichen Lehrstoffes und als Lernunterlage für Schüler/innen interpretiert. Tina Dworschak, Robert Vogler und Herbert Pichler plädierten für an die veränderten Rahmenbedingungen des kritischen kompetenzorientierten Lernens im digitalen Kontext angepasste Zukunftsmodelle. Pointiert formuliert steht dem welterklärenden Wissenskanon im Doppelseitenprinzip (ein Thema auf einer Doppelseite) inklusive angeschlossener Aufgaben ein multimedialer Zugriff gegenüber, der auf Problemzusammenhänge hinweist, exemplarisch Fragestellun-

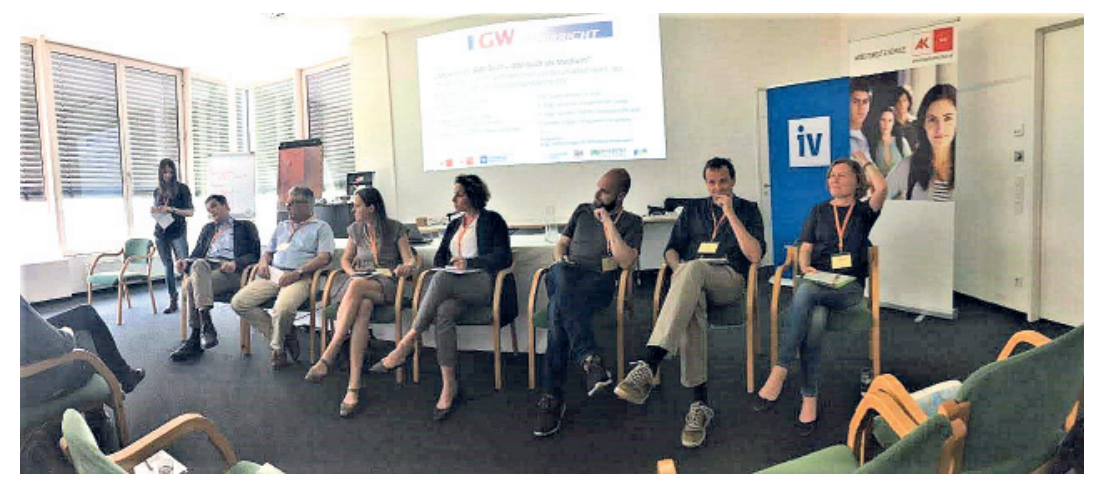

Abb. 7: Teilnehmer/innen der Podiumsdiskussion (Foto: Fridrich) 
gen und Themen aufarbeitet, methodische Zugänge erschließt, zum eigenen Forschen einlädt und zu originären und aktuellen Daten verlinkt. Dies geht freilich mit einem Bildungs- und Lernverständnis einher, das sich deutlich vom überwiegend reproduzierenden Charakter eines Schulunterrichts unterscheidet, der auch in verschiedenen aktuellen Schulbuchkonzepten nachgewiesen werden kann.

Einigkeit herrschte reihum bei der Kritik an der intransparenten Besetzungspolitik in Approbationskommissionen. Mehrere Diskutantinnen und Diskutanten verwiesen auf Untersuchungsergebnisse aus Schulbuchanalysen, die nachweisen können, wonach mehrere auf dem Markt befindliche Schulbücher nicht den jeweiligen Lehrplänen entsprechen und daher die Approbation nicht bekommen hätten dürfen. Da die Approbation in Österreich die Hauptinstitution der Qualitätssicherung für Schulbücher darstellt, wurde dieser Bereich mehrheitlich als Schlüsselproblem für die zukünftige Qualität der Schulbücher identifiziert.

Auch durch zahlreiche Redebeiträge aus den Reihen des Publikums von verschiedenen Lehrerinnen und Lehrern und Fachdidaktiker/innen entwickelte sich eine spannende, lebhafte und ergiebige Diskussion. Schlussendlich ginge es darum, so Richard Meisel, junge Menschen zu kritischen, entscheidungs- und handlungsfähigen Bürgerinnen und Bürgern zu erziehen. Bei der Diskussion darüber, mit welchem Medium dies am besten möglich sei, sollte dieses Ziel auf alle Fälle nicht aus den Augen verloren werden.

\subsection{Postersession}

Ein weiterer fixer Bestandteil der Fachdidaktiktagung ist die Young-Researcher-Poster-Session. Hierzu werden Nachwuchswissenschaftler/innen aus ganz

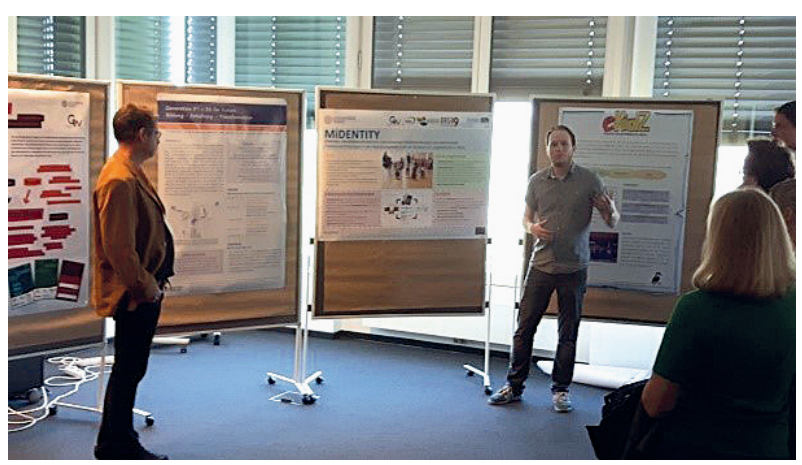

Abb. 8: Postersession (Foto: Pichler)

Österreich aufgerufen, ihre laufenden oder jüngst abgeschlossenen Forschungsarbeiten vorzustellen. In diesem Jahr reichte die Spannweite von präsentierten Bachelor-, Diplom- oder Masterarbeiten und Dissertationsprojekten bis hin zu Sparkling Science Projekten. Acht in Kürzestpräsentationen vorgestellte Poster dokumentieren, dass die Nachwuchsszene in der österreichischen Fachdidaktik wächst und beeindruckende Ergebnisse vorweisen kann. Die Poster sind auch in Zukunft aufzufinden (Tab. 1).

\subsection{Kabarett „Schöne neue Medienwelt“}

Das Künstlerduo Gerald Dell'mour und Rafael Wagner, die unter dem Künstlernamen „Geraf“" mittlerweile auch auf etablierten Kabarettbühnen auftreten, beleuchtete das Tagungsthema aus kabarettistischer Perspektive. Das temporeiche, speziell für die Veranstaltung zusammengestellte Programm machte auf unterhaltsame Weise deutlich, wie tief die wissenschaftlich und schulpraktisch diskutierten Fragen der neuen Kommunikations- und Medienwelten bereits im Lebensalltag von Kindern, Jugendlichen und Er-

Tab. 1: Übersicht über die Poster und ihren Speicherort

\begin{tabular}{|c|c|c|c|}
\hline Name(n) & Institution & Thema & Poster-Link \\
\hline Dalla-Via A. & $\begin{array}{l}\text { Karl-Franzens- } \\
\text { Universität Graz }\end{array}$ & Kartenspiel zum Thema, Global Cities'. & bit.ly/2LBQr7† \\
\hline Dolzer S. C. & $\begin{array}{l}\text { Alpen-Adria-Uni- } \\
\text { versität Klagenfurt }\end{array}$ & Wirtschaftswissen zum Thema ,Markt'. Ein Vergleich zwischen AHS und HAK. & bit.ly/2L2p7hl \\
\hline Golser K. & $\begin{array}{l}\text { Universität Salz- } \\
\text { burg }\end{array}$ & $\begin{array}{l}\text { Recht(sextrem)e Diskurse im Unterricht analysieren. Eine theoriegeleitete } \\
\text { Medienanalyse mit Schüler/innen. }\end{array}$ & bit.ly/2ktc5yu \\
\hline $\begin{array}{l}\text { Hintermann C., Pichler } \\
\text { H., Kessel V., Bergmeister } \\
\text { F. und Raithofer D. }\end{array}$ & Universität Wien & $\begin{array}{l}\text { MiDENTITY - (Mediale) Identitätskonstruktionen, transnationale Selbstver- } \\
\text { ortungen und verkürzende Fremdzuschreibungen in der Migrationsgesell- } \\
\text { schaft am Beispiel von Jugendlichen in Wien. }\end{array}$ & bit.ly/2IPsgoc \\
\hline $\begin{array}{l}\text { Lehner M. und } \\
\text { Stuppacher K. }\end{array}$ & $\begin{array}{l}\text { Universität Salz- } \\
\text { burg }\end{array}$ & $\begin{array}{l}\text { Suche nach dem G-Punkt. Queer inspirierte Praxis einer } \\
\text { schüler*innenorientierten Themenfindung für den GW-Unterricht. }\end{array}$ & bit.ly/2scVXoY \\
\hline $\begin{array}{l}\text { Parth S., Stötter H., } \\
\text { Keller L. }\end{array}$ & $\begin{array}{l}\text { Universität Inns- } \\
\text { bruck }\end{array}$ & eKidZ - Teach your parents well. & bit.ly/2JbirQZ \\
\hline Peduzzi D., Keller L. & $\begin{array}{l}\text { Universität Inns- } \\
\text { bruck }\end{array}$ & Generation F3 - Fit for Future. Bildung - Forschung - Transformation. & bit.ly/2JclhCB \\
\hline Prunner W. & Universität Wien & Civitas Nova. Ein Spiel für den Geographie und Wirtschaftskunde Unterricht. & bit.ly/2xo09aA \\
\hline
\end{tabular}


wachsenen verankert sind und wie sehr uns dies beeinflusst und oft auch überfordert. Im anschließenden Gespräch mit den Kabarettisten wurde erörtert, welcher Mehrwert sich durch eine humoristische oder kabarettistische Betrachtung von Themen auch in der Schule ergeben könnte. Satirische oder kabarettistische Aufgriffe können eine kritische Auseinandersetzung anregen und kommen dabei ohne den pädagogischen Zeigefinger aus.

\section{$4 \quad$ Feedback und Ausblick}

Wie hoffentlich aus dem Tagungsbericht ableitbar ist, haben wir eine konstruktive und ergebnisreiche Tagung erlebt, die von den Teilnehmerinnen und Teilnehmern in der abschließenden Evaluierung sehr positiv bewertet wurde. 89,7\% der Teilnehmer/innen benoten die Tagung als insgesamt sehr gut, die restlichen 12,3\% als gut, ähnlich hohe Zustimmungswerte fanden der Tagungsort, der Programmablauf und die Qualität der einzelnen Programmpunkte. Das Organisationsteam wird die Einzelrückmeldungen und Verbesserungsvorschläge im Detail besprechen und in die Organisation zukünftiger Tagungen einfließen lassen.

Der Termin für die Folgetagung ,Zukunft Fachdidaktik GW 2019' ist bereits fixiert: Von Montag 8. bis Mittwoch 10. April 2019 wird sie wiederum in Schlierbach unter dem Titel ,Konsum und Finanzen in der soziökonomischen Bildung' über die Bühne gehen. Dabei sollen Stakeholder, Fachdidaktiker/ innen, Schulpraktiker/innen und Studierende sozioökonomische Bildung zwischen wirtschaftlichen Zusammenhängen, Reflexivität, Kritikfähigkeit und Mitgestaltbarkeit von wirtschaftlichen Phänomenen, Strukturen und Prozessen bearbeiten und tragfähige Zukunftsperspektiven für die wirtschaftliche Bildung in GW ableiten. Es gibt bereits erste Zusagen namhafter Referentinnen und Referenten und Diskutantinnen und Diskutanten wie OeNB-Gouverneur Ewald Nowotny und Tim Engartner, Didaktiker der sozioökonomischen Bildung. Die Anmeldung zur Tagung als Fortbildungsveranstaltung der PH Linz ist bereits möglich. Ein detailliertes Programm und die Veröffentlichung der Vortragenden erfolgt Anfang 2019. Weitere Informationen finden Sie unter http:// gw.eduhi.at/FDtagungGW.

\section{Literatur}

Baecker, D. (2007): Studien zur nächsten Gesellschaft. Frankfurt am Main: Suhrkamp.

Bundesministerium für Bildung, Wissenschaft und Forschung (=BMBWF) (2017): Lehrpläne der AHS-Oberstufe. https://bildung.bmbwf.gv.at/schulen/unterricht/ lp/lp_ahs_oberstufe.html (30.5.2018)

Bundesministerium für Bildung, Wissenschaft und Kultur (=BMBWK) (2000): Lehrpläne der AHS-Unterstufe. Geographie und Wirtschaftskunde. https://bildung.bmbwf.gv.at/schulen/unterricht/lp/ahs9_784. pdf?61ebyf (30.5.2018) 